\title{
Participación parental, convivencia escolar y comunicación oral en estudiantes Peruanos
}

\section{Parental participation, school coexistence and oral communication in Peruvian students}

\author{
DOI: $10.46932 / \mathrm{sjjdv2n5-040}$
}

Received in: Jun 1st, 2021

Accepted in: Sep 30th, 2021

\author{
Delia Lidia Guerra-Condor \\ Mg. Administración de la Educación \\ Institution: Universidad César Vallejo \\ Address: Av. Alfredo Mendiola 6232, Los Olivos 15314 \\ E-maildelig07guerra@gmail.com \\ Abel Tapia Díaz \\ Dr. en Educación \\ Institution: Universidad César Vallejo \\ Address: Av. Alfredo Mendiola 6232, Los Olivos 15314 \\ E-mail atapiad@ucvvirtual.edu.pe \\ Jesús Emilio Agustín Padilla Caballero \\ PhD. Ética y Responsabilidad Social y Derechos Humanos \\ Institution: Universidad César Vallejo \\ Address: Av. Alfredo Mendiola 6232, Los Olivos 15314 \\ E-mailjpadillac@ucv.edu.pe \\ Maribel Cubas Diaz \\ Dr. en Educación \\ Institution: Universidad César Vallejo \\ Address: Av. Alfredo Mendiola 6232, Los Olivos 15314 \\ E-mail mfcdiaz12@gmail.com \\ Jenny Grados Moreno \\ Dr. en Educación \\ Institution: Universidad César Vallejo \\ Address: Av. Alfredo Mendiola 6232, Los Olivos 15314 \\ E-mail jennygrados25@gmail.com
}

\section{RESUMEN}

La presente investigación tuvo por objetivo determinar la influencia de la participación parental en la comunicación oral y la convivencia escolar en estudiantes de una institución educativa pública. Asimismo, el presente estudio se realizó dentro del enfoque cuantitativo, método hipotético deductivo, nivel explicativo y diseño no experimental; fue aplicado a 105 estudiantes de III ciclo de educación primaria y sus respectivos apoderados. Los instrumentos usados fueron dos cuestionarios para las variables Participación parental y Convivencia escolar, y una rúbrica para la variable Comunicación oral. Además, como resultados se obtuvo que las significancias son menores a 0,05 y el estadístico de Wall fue menor a 4, por lo tanto se llegó a la conclusión que la participación parental influye en la 
comunicación oral y convivencia escolar. Y según el estadístico de Nagelkerke la influencia de la variable participación parental sobre la convivencia escolar y comunicación oral es más del 30\%.

Palabras clave: participación de los padres; convivencia escolar; comunicación oral; expresión oral; relación padre-escuela.

\begin{abstract}
The present research aimed to determine the influence of parental participation in oral communication and school coexistence in students of a public educational institution. Likewise, the present study was carried out within the quantitative approach, hypothetical deductive method, explanatory level and nonexperimental design; it was applied to 105 students in the III cycle of primary education and their respective attorneys-in-fact. The instruments used were two questionnaires for the variables Parental participation and School coexistence, and a rubric for the variable Oral communication. In addition, as results it was obtained that the significance is less than 0.05 and the Wall statistic was less than 4 , therefore it was concluded that parental participation influences oral communication and school coexistence. And according to the Nagelkerke statistic, the influence of the parental participation variable on school coexistence and oral communication is more than $30 \%$.
\end{abstract}

Key words: parental participation; school coexistence; oral communication; oral expression; parentschool relationship.

\title{
1 INTRODUCCIÓN
}

A nivel mundial, a consecuencia de la Pandemia por la Covid19, el estado, los padres, docentes y estudiantes deben trabajar juntos, y desde su posición puedan ayudar con los aprendizajes de los estudiantes; por ello según el foro virtual organizado por la UNICEF (2020) se evidenció que la participación de los padres es indispensable en el aprendizaje virtual de los alumnos. Asimismo, según la BBC News (2020) menciona que los padres han llegado a ser docentes auxiliares que apoyan a sus hijos a resolver trabajos, escaneando o grabando videos del proceso de desarrollo de las evidencias de aprendizaje.

Por otra parte, la comunicación es un medio oral y surge con la existencia del hombre, siendo una capacidad propia gracias a sus relaciones sociales. A través de la comunicación, el ser humano puede expresarse con otros individuos y su comunidad, de forma creativa y debe asumir su responsabilidad por lo dicho. Además, la comunicación oral favorece la identidad del individuo y su desarrollo personal (Minedu, 2017).

En la actualidad, el saber expresarse de manera oral es prácticamente una necesidad, presente en exposiciones orales, relaciones familiares, clases y comunicaciones virtuales. Para (Cassany, Luna y Sanz, 2008) en la infancia y luego la niñez donde se establecen las bases de la comunicación, de ahí radica la importancia de la estimulación del núcleo familiar. Así también, el vínculo con los agentes educativos asegura niveles de logro aceptables en los aprendizajes. 
Por otro lado, la convivencia escolar son relaciones entre los seres humanos en un determinado contexto escolar, que obedece al respeto por los derechos humanos y favorece el desarrollo integral y el logro de los aprendizajes (Minedu, 2018). Por lo tanto, si se pretende que se consolide el aprendizaje y se fortalezca la formación integral de los estudiantes, se debe asegurar un ambiente favorable de convivencia estudiantil que genere relaciones interpersonales respetuosas y satisfactorias.

Lo expuesto con anterioridad, guarda estrecha relación con lo observado en la institución educativa N ${ }^{o}$ 20955-13 Paulo Freire de Huarochirí donde los estudiantes presentan dificultades en la comunicación oral tal como: repiten las mismas palabras, tiemblan, sudan, se traban y también, muchos de ellos tienen dificultad para pronunciar adecuadamente las consonantes como r y z, y las sílabas trabadas.

Por otro lado, la presente investigación tiene como objetivo determinar el nivel de influencia de la participación parental sobre la convivencia escolar y comunicación oral. Así también, la hipótesis general es que: la participación parental influye en la comunicación oral y convivencia escolar, y las hipótesis específicas que las dimensiones vinculares, formativas, protectoras y reflexivas influyen en la comunicación oral y la convivencia escolar.

La justificación práctica, está en la importancia que tienen los padres al estar involucrados en la formación de sus hijos que es una solución real a la problemática que se presentó en la presente investigación. Además, como justificación metodológica, se tiene la validación de instrumentos de las variables siendo de relevancia para otros investigadores que pueden utilizar y aplicar en otro contexto educativo. Finalmente en el plano psicológico, se justifica porque la participación parental es vital en la identidad del ser humano.

Con la finalidad de mejorar los aprendizajes de los estudiantes se busca que los padres participen en la formación de sus hijos. Además en la presente investigación se buscó antecedentes, bases teóricas y dimensiones de las variables de estudio, asimismo los instrumentos han sido validados por juicio de expertos, con el fin de determinar la influencia de la participación parental en la comunicación oral y la convivencia escolar. El cual se determinará el nivel de influencia de los padres en la formación holística de sus hijos.

\section{MARCO TEÓRICO}

Como antecedentes internacionales, se tiene a Domeniconi y Gràcia (2018) y Gómez (2017) quienes implementaron una intervención enfocada en la familia. Se contó con la participación de cuatro familias, donde al menos un hijo tenía cinco años. Como instrumentos se usaron la Prueba de Lenguaje Oral Navarra (PLON), y la escala de valoración de la lengua oral en contexto familiar (Escala EVALOF). Se concluyó que los padres desaprovechan las rutinas diarias para hablar con sus hijos. En este sentido se 
rescata la importancia de la interacción comunicativa en la familia y el uso de cuentos como rutina para propiciar el desarrollo del lenguaje.

Así también, Jiménez (2019) buscó incorporar tácticas educativas para la comunicación verbal, en el entorno del Reajuste Curricular, usó la herramienta del trabajo de campo para la recolección de información tuvo como muestra a 25 alumnos de décimo año en Quito. Los resultados demuestran que el sentido comunicativo es necesario para el desarrollo de la enseñanza porque considera las necesidades del estudiante.

Además, Vargas, Morales, Witto, Zamorano, Olhaberry y Farkas (2016) investigaron la influencia de la mentalización de los cuidadores principales y el nivel socioeconómico sobre el nivel de desarrollo del lenguaje alcanzado en un grupo de 85 niños y niñas a los 2 años y medio de edad. Se concluye que el lenguaje es una competencia infantil sobresaliente pues se vincula con diferentes aspectos de la vida del niño y con el nivel socioeconómico.

Asimismo, el estudio que realizó Suárez (2017) sobre la asociación de la participación parental con la convivencia escolar, se buscó determinar la influencia que ejerce el papel de los padres en el entorno de convivencia escolar para el estudiante, la muestra fueron niños de 4 y 5 años. Se concluyó que el papel de padre no es cumplido en su totalidad, esta figura se muestra ausente y permisiva. Además, Pérez y Santillán (2018) realizaron un estudio de tipo descriptivo donde se estudió la convivencia de los niños de 5 y 6 años respectivamente en la escuela. En esta investigación se llegó a la conclusión que los aprendizajes académicos influyen la manera de pensar y actuar; pero las diversas costumbres y los patrones de crianza pueden provocar indicadores negativos en los niños.

Así también, se tiene el estudio de Bowie y Ward (2018) quienes propusieron la estructura y las pautas de funcionamiento de una Escuela para Padres con el fin de ayudar a la formación escolar y la mejora de la convivencia escolar para niños de primaria. Los resultados muestran que la escuela debe cumplir un rol motivador para el docente, para que cumpla con una buena función educativa y llegue al núcleo familiar. De forma similar, Suastegui y Mojica (2019) buscaron soluciones para mejorar el entorno familiar con relación a la convivencia escolar de los estudiantes en una población de 118 personas: docentes, estudiantes y representantes legales. Del estudio se obtuvo que, los padres son el primer núcleo formador de valores y habilidades sociales.

Mientras que, Barrios, Marrero, Gómez y Quiroz (2019) aplicaron un plan de acción dirigido a fortalecer la convivencia y la comunicación en una institución educativa pública en Venezuela. Los resultados muestran que la comunicación es la opción ideal para mejorar la convivencia escolar porque influye en la resolución de problemas y también los previene en base al diálogo, el respeto y la tolerancia. 
Por otro lado, el estudio de Velásquez (2019) quien buscó determinar el nivel de sobreprotección de los padres y su influencia en el desarrollo social de los niños. Legó a conclusión que, sí existe un alto nivel de sobreprotección de los padres e influyen poderosamente en el desarrollo de los niños. Así también, para Palomino (2017) que estudió la participación parental. El estudio de diseño cuasi experimental de tipo documental estuvo conformado por una muestra de 39 integrantes. Los resultados mostraron que el programa de sensibilización a los padres contribuye de forma positiva en el desarrollo de la comunicación de los alumnos.

Asimismo, Luna, Montealegre y Orozco (2019) estudiaron los pensamientos y sensaciones de madres adolescentes y sus bebés. Se concluyó que el papel de la madre es único en el proceso comunicativo del niño, ella usa habilidades propias para favorecer el vínculo y es capaz de cambiar su comportamiento en busca de adaptación a la necesidad del niño. Asimismo, De la Cruz (2019) realizó un estudio sobre las actitudes de las madres y el lenguaje oral en 54 niños de 5 años de edad. Se concluyó que las actitudes maternas son de mayor dominio sobre indulgencia y sobreprotección. Pero también que la relación madre-hijo es valiosa cuando se genera en un ambiente sano de aceptación cuando esta permite experimentar al niño, lo cual produce un mayor desarrollo del lenguaje oral. Del mismo modo, Navarro (2018) estudió la influencia de la familia en la expresión oral. Se concluyó, que el 69,9\% de los padres afirman que la comunicación entre hermanos se da con mayor facilidad, 56\% de los alumnos en el aula usan el mismo vocabulario que usan en casa.

Además, Flores (2018) investigó sobre el impacto de un programa educativo en la convivencia escolar de una población de 50 estudiantes dividida en dos grupos: la sección "A” grupo experimental y la sección "B" grupo control. Los resultados evidencian que en el desarrollo educativo juegan un papel muy importante los aspectos corporales, emocionales y racionales como una unidad inseparable y complementaria, sobre la cual se construyen los aprendizajes y el bienestar humano.

Luego de la revisión de antecedentes se presenta las bases teóricas de cada una de las variables. La Participación parental. Alude a todas las labores que cumplen los padres para el desarrollo psicológico y cognitiva de sus hijos en el hogar o fuera de este. Asimismo, los padres velan por cuidar la salud y bienestar, alimentación, ropa, casa y educación de sus hijos González (2018). La familia es la primera institución que ejerce influencia en el niño, transmite valores, costumbres y creencias por medio de la convivencia diaria. Además, es la primera institución educativa y socializadora del niño, pues desde su nacimiento está sujeto a la influencia del entorno familiar Guevara (1996). Aquí se observa la importancia de la familia como constructora del cimiento cultural sobre los hijos en quienes, claramente, deja marcas perdurables. 
Para Barudy, Dantagnan (2010) definen las competencias parentales como el resultado de la relación entre las condiciones psicosociales de la familia, el escenario educativo que los apoderados han construido y las características del hijo. Como se observa, la participación parental nace y se desarrolla dentro del ambiente familiar, y está directamente asociada al rendimiento académico de los alumnos. Asimismo, el ambiente familiar es el grupo de vínculos que se rigen entre los integrantes de una familia que comparten el mismo ambiente.

Asimismo, Navarro (2018) y (Barudy y Dantagnan, 2005) afirman que, el ambiente familiar influye de forma determinante en la identidad del ser y su comportamiento. Por ello el niño adquiere desde su nacimiento los afectos, actitudes, valores y enseñanzas que observa y vive de la relación con otros miembros de su hogar. Además, el autor citado resalta la familia como el sistema de socialización más influyente, fundamental y el que permanece a lo largo de la vida del individuo.

Para Gómez y Muñoz (2014) quienes diseñaron la Escala de Parentalidad (e2p) basándose en la teoría ecosistémica del desarrollo humano, la teoría del apego y la resiliencia humana por ser un estudio que recoge todas esas impresiones anteriores, las confronta y adapta; a su vez, consideran las competencias parentales como un conjunto de conocimientos y capacidades que conducen el actuar parental en distintas situaciones y/o necesidades comunicativa, física, socioemocional y cognitiva que implica la crianza de un hijo a fin de garantizar el bienestar y sus derechos.

Para Gómez y Muñoz (2014) establece las dimensiones: vinculares, son aquellas que buscan el apego seguro y un óptimo desarrollo socioemocional; esto, comprenden una serie de comportamientos de orden visual, oral, anímico o corporal que los padres emplean para promover cambios en sus hijos. Formativas, son aquellas que favorecen el desarrollo, el aprendizaje y la socialización de los hijos; a través de conductas, saberes y destrezas parentales. Protectoras, busca el cuidado y la protección de los niños para satisfacer sus necesidades y garantizar el cumplimiento de sus derechos, de esta manera, se estaría resguardando la integridad física, sexual y emocional. Y reflexivas, que busca la reflexión y monitoreo sobre el papel parental sobre los hijos, así como la evaluación del desarrollo integral de estos, de esta manera, esta dimensión ayuda, cual complemento, las otras dimensiones.

En relación, a la comunicación oral para Aizpún (2013)) afirma que: el lenguaje es propio del género humano que exige una actividad equilibrada y armónica de factores biológicos, psicológicos y ambientales. En ese sentido, el lenguaje como forma propia del individuo se percibe desde la gestación y nos acompaña a lo largo de nuestra vida, y está presente en cada etapa del desarrollo del ser. Para (Torán, 2016). El lenguaje es la capacidad y el medio de comunicación que posee el ser humano que le permite la interacción social, medio por el cual puede expresar y percibir sentimientos e ideas. 
Asimismo, el lenguaje concede la posibilidad de comunicación y refuerza la pertenencia a un determinado grupo social donde permite la interrelación con los demás (Suybate, 2019) y Torán (2016). Y para Perez y Salmeron (2006) afirmando que el lenguaje es el instrumento de comunicación propio del ser humano que sirve para obtener, ordenar, dirigir el pensamiento y la propia acción, además de ser el recurso más complejo y completo aprendido naturalmente por una serie de intercambios con el medio ambiente a través de otros interlocutores más competentes.

Mientras que, para Ramírez (2002) la comunicación oral consiste en el dominio de habilidades comunicativas de lenguaje oral que van más allá de la interpretación y emisión de los sonidos organizados en signos lingüísticos que obedecen a la gramática. En este sentido, la comunicación oral está presente en los discursos diarios, en el trabajo, en el juego, en el plano comercial, amoroso, emocional, entre otros. La imagen del emisor y receptor, así como el espacio que ocupan, la vestimenta, ornamentación, la expresión del cuerpo, los gestos, la modulación de voz, la velocidad al hablar, las sonrisas, entre otros, son parte complementaria de la comunicación oral.

También, es la interacción entre uno o más interlocutores a fin de expresar y comprender emociones e ideas, los cuales hacen de hablantes u oyentes (Arapa y Cuadros, 2018). Por este motivo, se afirma que es una práctica social donde el estudiante hace uso del lenguaje oral de forma creativa, en ese camino, constituye su identidad y favorece su desarrollo personal.

Asimismo, las dimensiones de la comunicación oral según (Arapa y Cuadros, 2018) son: obtiene información, donde el estudiante es capaz de recuperar y extraer información evidente expresada por los participantes (Arapa y Cuadros, 2018). Infiere e interpreta, donde se construye el sentido del texto al relacionar información evidente y no evidente a partir de recursos verbales, y no verbales (Arapa y Cuadros, 2018). Adecúa y organiza, donde el estudiante manifiesta sus ideas según el propósito comunicativo y destinatario, (Arapa y Cuadros, 2018). Utiliza recursos no verbales, el estudiante usa recursos no verbales, gestos o movimientos corporales (Arapa y Cuadros, 2018). Interactúa, se caracteriza porque el rol del estudiante es cambiante (Arapa y Cuadros, 2018). Y Reflexiona, en esta capacidad se observa la relación entre los procesos de reflexión y evaluación (Arapa y Cuadros, 2018).

En relación con la variable Convivencia escolar, Barrios, Marrero, Gómez y Quiroz (2019) la definen como una cohabitación con otros donde es importante establecer condiciones; también aseguran que es un proceso que consiste en el reconocimiento del otro, por ello la comunicación juega un papel muy importante en este convivir. Del mismo modo, Carretero (2008) asegura que es el proceso donde los miembros de una comunidad educativa aprenden a vivir con otras personas.

Por otro lado, en el ámbito pedagógico la convivencia escolar requiere de la comunicación entre los agentes educativos con la finalidad de crear una atmosfera adecuada que imparta seguridad Morales y 
Rodríguez (2017). Además, la convivencia es una pieza fundamental para el desarrollo escolar, Manriquez (2014) y Sandoval (2014); es decir es necesario convivir en armonía sobre la base del respeto y tolerancia a ideas y sentimientos de los otros.

Por lo tanto, la convivencia se ha convertido en un elemento protagónico en el proceso educativo, la escuela debe crear vínculos armoniosos para practicar la convivencia sustentados en el respeto hacia los demás. Para Fernández (2015); Saneleuteiro y López (2017) afirman que el término convivencia escolar se emplea en el sector educación para reducir la estadística de violencia al interior de las escuelas. Al abordar esta variable Challco (2018) determina que es el conjunto de relaciones que se llevan a cabo en las escuelas, siendo su cuidado y responsabilidad de todos los miembros de la comunidad educativa y que tiene por objetivo el vivir democráticamente.

Finalmente, Challco (2018) establece como dimensiones de la convivencia escolar: la primera, interactúa, el estudiante muestra preocupación por los demás y respeta las diferencias como parte de su aprendizaje; para interactuar con otros sobre la base del respeto se requiere de control comunicativo Challco (2018). La segunda, construye normas, que consiste en que el estudiante forma parte de quienes construyen las normas que se aplicarán en su institución, haciendo un proceso de evaluación de estas y respetando su ejecución Challco (2018); la tercera, maneja conflictos, aquí se observa que el estudiante es capaz de resolver problemas de forma creativa y empática Duárez (2019); y Challco (2018). La cuarta, delibera, el estudiante se involucra en asuntos que conciernen a todos y participa del diálogo donde expone sus ideas Duárez (2019) y Challco (2018). La quinta, participa, el estudiante usa canales de participación democrática para liderar iniciativas de interés de la comunidad educativa donde se practiquen y defiendan los derechos humanos Challco (2018).

\section{MÉTODO}

La presente investigación es de tipo básica, busca probar una teoría sin intención de aplicar los resultados a problemas prácticos Hernandez-Sampieri y Mendoza (2018), en ese sentido, solo se efectuó la búsqueda de nuevos conocimientos y teorías. El diseño es no experimental debido a que los fenómenos han sido observados y analizados en su estado natural sin manipulación de las variables HernandezSampieri y Mendoza (2018). Asimismo, el enfoque fue cuantitativo porque tuvo por objetivo la medición y el cálculo de la magnitud de las variables a través de la recolección y análisis de datos. Por otro lado, el nivel en el que se halla este estudio es el explicativo, el cual implicó más que una descripción o relación entre fenómenos o conceptos fue la explicación de las causas Hernandez-Sampieri y Mendoza (2018).

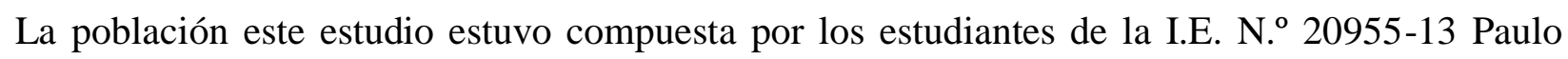
Freire de Huarochirí, siendo 253 estudiantes del nivel primaria, la muestra es un fragmento representativo 
de la población (Niño, 2011). En este caso, la muestra estuvo compuesta por los estudiantes del ciclo III del nivel primaria, conformado por $1^{\circ}$ y $2^{\circ}$ grado siendo en total 105 estudiantes. Se eligió esta muestra debido que es el ciclo donde se inicia el nivel primaria y es la base de todos los demás ciclos y grados.

La técnica usada en esta investigación fue la encuesta, la cual sirve para recoger datos referidos al estudio a través de interrogantes (López-Roldán y Fachelli, 2015), esto fue para las variables participación parental y convivencia escolar, la observación fue la técnica para el caso de la variable comunicación oral, que consiste en un registro sistemático de comportamientos. Asimismo el instrumento que se usó en esta investigación fue el cuestionario para la participación parental y convivencia escolar. Y para la comunicación oral fue la rúbrica.

Para la validez se realizó la prueba de Aiken, cuyos coeficientes fueron mayores a 0,70. Además, el presente instrumento fue validado por cinco expertos. Además, para la confiabilidad de los instrumentos se aplicó el Alpha de Cronbach cuyos resultados fueron para participación parental 0,979. Para convivencia escolar fue 0.847 y para comunicación oral fue 0,871 . De esta manera, se ha demostró que los instrumentos son confiables y válidos.

\section{RESULTADOS}

A continuación, se presentan los resultados del procesamiento de los datos inferenciales.

Tabla 1. Estimaciones de parámetro, Seudo R cuadrado y ajuste de modelo

\begin{tabular}{|c|c|c|c|c|c|c|c|c|c|}
\hline \multirow{4}{*}{ 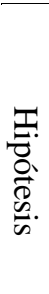 } & \multirow{4}{*}{$\begin{array}{l}\text { Convivencia = Alto } \\
\text { Comunicación } \\
\text { oral=Inicio } \\
\text { Comunicación oral } \\
=\text { logro-prev }\end{array}$} & \multirow{2}{*}{$\begin{array}{l}\text { Wald } \\
4,020\end{array}$} & \multirow{2}{*}{$\begin{array}{l}\mathrm{gl} \\
1\end{array}$} & \multirow{2}{*}{$\begin{array}{l}\text { Sig. } \\
, 042\end{array}$} & \multirow{2}{*}{$\begin{array}{c}\text { L.Inf. } \\
-51250,061\end{array}$} & \multirow{2}{*}{$\begin{array}{c}\text { L.Sup. } \\
51206,604\end{array}$} & \multirow{2}{*}{$\begin{array}{c}\text { Seudo R2 } \\
\text { Cox y Snell 0,061 }\end{array}$} & \multicolumn{2}{|c|}{ Ajuste de modelo } \\
\hline & & & & & & & & Sig &, 020 \\
\hline & & 45,456 & 1 &, 000 & $-18,082$ & 18,082 & Nagelk. 0,353 & Gl & 4 \\
\hline & & 32,45 & 1 &, 000 & $-51248,269$ & 51208,396 & Mc Fadden 0,332 & $\begin{array}{l}\text { Chi- } \\
\text { cuadrado }\end{array}$ & 6,339 \\
\hline \multirow{3}{*}{ 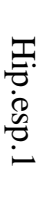 } & \multirow{3}{*}{$\begin{array}{l}\text { Convivencia =alto } \\
\text { Comunicación } \\
\text { oral=Inicio } \\
\text { Comunicación oral } \\
=\log \text {-previsto }\end{array}$} & 5,52 & 1 &, 045 & $-18,082$ & 18,082 & Cox y Snell 0,059 & Sig &, 018 \\
\hline & & 12,52 & 1 & ,035 & $-51248,269$ & 51208,396 & Nagelk. 0,341 & Gl & 4 \\
\hline & & 5,52 & 1 & ,030 & $-54021,634$ & 54022,141 & Mc Fadden 0,320 & $\begin{array}{l}\text { Chi- } \\
\text { cuadrado }\end{array}$ & 6,529 \\
\hline \multirow{3}{*}{ 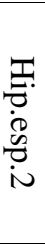 } & \multirow{3}{*}{$\begin{array}{l}\text { Convivenvia }= \\
\text { medio] } \\
\text { comunicación oral } \\
\text { =log-previsto }\end{array}$} & 23,320 & 1 & ,015 & $-18,082$ & 18,082 & Cox y Snell 0,062 & Sig &, 015 \\
\hline & & 34,300 & 1 & 0,010 & $-54021,634$ & 54022,141 & Nagelk. 0,358 & Gl & 4 \\
\hline & & & & & & & Mc Fadden 0,337 & $\begin{array}{c}\text { Chi- } \\
\text { cuadrado }\end{array}$ & 6,674 \\
\hline \multirow{3}{*}{ 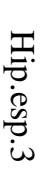 } & Convivencia $=$ Bajo & 484,693 & 1 &, 000 & $-51250,061$ & 51206,604 & Cox y Snell 0,064 & Sig &, 014 \\
\hline & Convivencia $=$ alto & 32,000 & 1 & ,002 & $-19,765$ & 19,765 & Nagelk. 0,371 & Gl & 4 \\
\hline & $\begin{array}{l}\text { Comunicación } \\
=\text { logro prev }\end{array}$ & 6,024 & 1 &, 025 & $-51248,269$ & 51408,396 & Mc Fadden 0,349 & $\begin{array}{c}\text { Chi- } \\
\text { cuadrado }\end{array}$ & 6,917 \\
\hline$\Xi$ & $\begin{array}{l}\text { Convivencia }= \\
\text { proceso }\end{array}$ & 464,591 & 1 &, 017 & $-51633,09$ & 51589,612 & Cox y Snell 0,058 & Sig & ,018 \\
\hline
\end{tabular}


Comunicación $=$

inicio

$196,944 \quad 1 \quad, 012$

$-51631,30$

51591,403

Nagelk. 0,335

Comunicación

5,500

$-51630,20$

51592,502

Mc Fadden 0,314

Chi-

$=$ proceso

$1 \quad, 043$

La Hipótesis General es: la participación parental influye en la comunicación oral y la convivencia escolar en estudiantes de una institución educativa Pública, de la tabla los resultados mostrados se tiene las estadísticas de estimación del parámetro y se muestra la variable predictora, participación parental incide significativamente en las variables comunicación oral y convivencia escolar. Se muestra los coeficientes de Wald son 0.042 y 0.00 son significativos en convivencia escolar en el nivel alto y comunicación oral en el nivel inicio y logro previsto.se tiene que el p-valor de la prueba es menor que 0.05 , por lo que se rechaza la hipótesis nula. Asimismo, se tiene que el valor de Nagelkerke es de 0,353 indica que el $35.3 \%$ de la variabilidad de las variables comunicación oral y convivencia escolar es explicado por la participación parental.

La dimensión específica una. La dimensión vincular influye en la comunicación oral y convivencia escolar, de los datos de la tabla, se tiene que el p-valor de la prueba es menor que 0.05 , por lo que se rechaza la hipótesis nula. Además, se muestra la dimensión predictora, vincular que incide significativamente en las variables comunicación oral y convivencia escolar. Se muestran los coeficientes de Wald son 0.45 y 0.035 son significativos en convivencia escolar en el nivel adecuado y comunicación oral en el nivel inicio. Asimismo, el valor de Nagelkerke es de 0,341 indica que el 34.1\% de la variabilidad de las variables comunicación oral y convivencia escolar es explicado por la participación parental.

La hipótesis especifica dos. La dimensión formativa influye en la comunicación oral y convivencia escolar en estudiantes, de los resultados mostrados se tiene las estadísticas de estimación del parámetro y se muestra la dimensión predictora, formativa incide significativamente en las variables comunicación oral y convivencia escolar dado que los coeficientes de Wald son 0.15 y 0.010 son significativos en convivencia escolar en el nivel medio y comunicación oral en el nivel logro previsto. Asimismo, el pvalor de la prueba es menor que 0.05 , por lo que se rechaza la hipótesis nula. Además, el valor de Nagelkerke es de 0,358 , lo cual indica que el 35,8\% de la variabilidad de las variables comunicación oral y convivencia escolar es explicado por la dimensión formativa.

La hipótesis específica tres. La dimensión protectora influye en la comunicación oral y convivencia escolar, de los resultados mostrados la dimensión predictora, protectora incide significativamente en las variables comunicación oral y convivencia escolar dado que los coeficientes de Wald son 0.02 y 0.025 son significativos en convivencia escolar en el nivel alto y comunicación oral en el nivel logro previsto. Además, el p-valor de la prueba es menor que 0.05, por lo que se rechaza la 
hipótesis nula. Y el valor de Nagelkerke es de 0,371 indica que el 37,1\% de la variabilidad de las variables comunicación oral y convivencia escolar es explicado por la dimensión protectora.

La hipótesis especifica cuatro. La dimensión reflexiva influye en la comunicación oral y convivencia escolar. De los resultados mostrados se tiene las estadísticas de estimación del parámetro y se muestra la dimensión predictora, reflexiva incide significativamente en las variables comunicación oral y convivencia escolar. Se muestras los coeficientes de Wald son 0.017 y 0.012 son significativos en convivencia escolar en el nivel proceso y comunicación oral en el nivel inicio y proceso. De los datos de la tabla, respecto a la información del modelo, dado que el p-valor de la prueba es menor que 0.05, se rechaza la hipótesis nula. Por lo tanto, existen evidencias estadísticas para indicar que el modelo con las variables trabajadas influye en el ajuste de forma significativa. De la tabla del $\mathrm{R}$ pseudo cuadrado se tiene que, el valor de Nagelkerke es de 0,335 indica que el 33,5\% de la variabilidad de las variables comunicación oral y convivencia escolar es explicado por la dimensión reflexiva.

\section{DISCUSIÓN}

Producto de los hallazgos del capítulo de resultados, se tiene que la variable participación parental influye en un 35,3\% en las variables comunicación oral y convivencia escolar. En este sentido, Gómez (2017) plantean una serie de factores que influyen en la convivencia escolar como el institucional donde entra a tallar las características de la dirección y la gestión de la institución; también la organización interna relacionado con la forma en que las personas se organizan y coordinan para realizar funciones diarias. Para Suárez (2017) sostiene que la convivencia escolar gira en torno a lo familiar, social, emocional, los cuales se relacionan entre sí y son capaces de afectar de manera positiva o negativa a la institución educativa, por esto, es necesario involucrar a cada miembro de la comunidad con el fin de generar la interrelación y crear ambientes adecuados para la convivencia escolar. Además, Barrios, Marrero, Gómez y Quiroz (2019) mencionaron sobre la importancia de la comunicación, pues esta se perfila como la opción ideal para mejorar la convivencia escolar porque influye en la resolución de problemas.

Por otro lado, sobre los resultados de la primera hipótesis específica se demostró que, la dimensión vincular influye en la comunicación oral y convivencia, cuya incidencia de la dimensión es del 34\% en los estudiantes de una institución pública. La dimensión vincular busca el apego seguro y un óptimo desarrollo socioemocional (Gómez y Muñoz, 2014). El ambiente familiar es el grupo de vínculos que se rigen entre los integrantes de una familia que comparten el mismo ambiente. Todas las familias son diferentes, por ende, viven y participan en las relaciones de forma única lo que las diferencia entre sí. Complementa Navarro (2018) el ambiente familiar influye de forma determinante en la identidad del ser 
y su comportamiento. El niño adquiere desde su nacimiento los afectos, actitudes, valores y enseñanzas que observa y vive de la relación con otros miembros de su hogar.

La segunda hipótesis específica se demostró que, la dimensión formativa influye en la comunicación oral y convivencia, cuya incidencia de la dimensión es del 35.8\% en los estudiantes de una institución pública. Para Suastegui y Mojica (2019) quienes afirman que, los padres son el primer núcleo formador de valores y habilidades sociales; y, en el campo escolar, los docentes están dispuestos a reforzar el tema de valores humanos, empatía e interacción para una convivencia escolar positiva. Así como para Perez y Salmeron (2006) afirmaron que el lenguaje es el instrumento de comunicación propio del ser humano que sirve para obtener y pasar información, ordenar y dirigir el pensamiento y la propia acción, además de ser el recurso más complejo y completo aprendido de manera natural. Para (Gómez y Muñoz, 2014). La participación parental influye de forma determinante en la identidad del ser y su comportamiento. Y (Navarro, 2018) concluye que la familia es el sistema de socialización más influyente y fundamental en los resultados instruccionales, formativos y afectivos.

De la tercera hipótesis específica se demostró que, la dimensión protectora influye en la comunicación oral y convivencia, cuya incidencia de la dimensión es del 37.1\% en los estudiantes de una institución pública. Al respecto, la dimensión protectora busca proteger a los niños y cuidar de estos para satisfacer sus necesidades y garantizar el cumplimiento de sus derechos, de esta manera, se estaría resguardando la integridad física, sexual y emocional (Gómez y Muñoz, 2014). Sobre esta dimensión, Velásquez (2019) afirmó que, al haber un alto nivel de sobreprotección de los padres, esto influye poderosamente en el desarrollo de los niños por ello se recomienda inculcarles autonomía, independencia y una educación en valores sin perder el afecto y la buena comunicación.

De la cuarta hipótesis específica se demostró que, la dimensión reflexiva influye en la comunicación oral y convivencia, cuya incidencia es del 33.5\% en los estudiantes de una institución pública. Al respecto, la dimensión reflexiva busca la autorreflexión y automonitoreo del papel parental sobre los hijos y el autocuidado de sí mismos, de esta manera, para (Gómez y Muñoz, 2014) la participación parental está compuesta por capacidades que son propias, innatas y esenciales para el ser, sin embargo, ajustadas por las experiencias vividas e influenciadas por el entorno educativo y social. Además, para (Barudy y Dantagnan, 2005), la reflexión que hacen los padres sobre su propio papel de padres y cómo esto viene afectando sus vidas es importante para ocuparse de la formación de sus hijos.

\section{CONCLUSIONES}

Primera. La participación parental influye en la comunicación oral y la convivencia escolar, cuya incidencia de la variable independiente es del $35,1 \%$ en los estudiantes de una institución pública. Es 
recomendable abordar la variable participación parental generando un compromiso de ayuda de parte de los padres a sus hijos al inicio de un proyecto de investigación, de esa manera, se podría complementar el presente estudio y dar luces del contexto real en el que se desenvuelven los estudiantes de la institución educativa motivo de estudio.

Segunda. La dimensión vincular influye en la comunicación oral y convivencia, cuya incidencia de la dimensión es del $34 \%$ en los estudiantes de una institución pública. Pese a que se han hallado resultados favorables en la variable convivencia escolar, es recomendable que los docentes identifiquen a los principales estudiantes promotores de dicha convivencia con la finalidad de hacer un efecto multiplicador entre pares.

Tercera. La dimensión formativa influye en la comunicación oral y convivencia, cuya incidencia de la dimensión es del 35.8\% en los estudiantes de una institución pública. No se debe perder de vista los resultados en Comunicación oral que alcanzó esta investigación, en este sentido, se deben plantear estrategias que refuercen la competencia ya que sus valores numéricos (notas) indican que los estudiantes se encuentran mayormente en el nivel "En proceso".

Cuarta. La dimensión protectora influye en la comunicación oral y convivencia, cuya incidencia de la dimensión es del 37.1\% en los estudiantes de una institución pública. Se debe hacer énfasis en la relación de los padres con sus hijos de tal manera que la comunicación entre ellos sea fluida, se debe adicionar en las investigaciones talleres de manejo efectivo de la comunicación.

Quinta. La dimensión reflexiva influye en la comunicación oral y convivencia, cuya incidencia de la dimensión es del 33.5\% en los estudiantes de una institución pública. Es necesario que en la escuela se establezca taller a los padres de familia para que puedan generarse espacios de reflexión de los padres, de tal manera que se les comprometa en su rol de padres orientadores y ahora como docentes en pandemia. 


\section{REFERENCIAS}

Aizpún, T. (2013). Dialéctica y Libertad. Tópicos, 7(1), 33-42. https://doi.org/10.21555/top.v7i1.488

Arapa, E. y Cuadros, V. (2018). Influencia del uso de la aplicación web duolingo en la capacidad de comprensión de textos orales en inglés de los estudiantes de primer grado de secundaria de la institución educativa "Héroes del pacifico" de la provincia de Arequipa 2018 (tesis de Posgrado). Universidad Nacional de San Agustín de Arequipa, Perú.

Barrios, N., Marrero, E., Gómez, M. y Quiroz, J. (2019). Convivencia y comunicación escolar: Transformación desde la acción y la participación. INNOVA Research Journal, 4 (3.1), 89-107. doi: /10.33890/innova.v4.n3.1.2019.1056

Barudy, J. y Dantagnan, M. (2005). Los buenos tratos a la infancia. Parentalidad, apego y resiliencia. Barcelona: Gedisa.

Barudy, J. y Dantagnan, M. (2010). Los desafíos invisibles de ser padre o madre: Manual de evaluación de las competencias y la resiliencia parental. Recuperado de https://books.google.com.pe/books?hl=es\&lr=\&id=PyUlBQAAQBAJ\&oi=fnd\&pg=PA15\&dq=barudy+ 2010+concepto+parentalidad\&ots=Hc0_8I5tiM\&sig=AaT0gdawJuF2EHvXZc93alpskvo\#v=onepage\&q =barudy\%202010\%20concepto\%20parentalidad $\& \mathrm{f}=$ false

Bowie, L. y Ward, B. (2018). Escuela para padres como vía de fortalecimiento de la convivencia escolar (tesis de posgrado). Universidad de la Costa CUC, Colombia.

Cassany, D., Luna, M. y Sanz, G. (2008). Las cuatro destrezas: expresión oral. Recuperado de https://www.quiben.net/procesos-infantil/wp-

content/uploads/2017/02/las_cuatro_destrezas_expresin_oral.pdf

Challco, D. (2018). “Aplicación de juegos tradicionales para promover competencias de convivencia y participación democrática en los alumnos del IV ciclo I.E. $N^{\circ} 50756$ San Antonio del distrito de Echarati - La Convención" (tesis de posgrado). Universidad Cesar Vallejo, Perú.

De la Cruz, B. (2019). Actitudes maternas y lenguaje oral en estudiantes de cinco años de una institución educativa pública del Callao (tesis de pregrado). Universidad San Ignacio de Loyola, Perú.

Domeniconi, C. y Gràcia, M. (2018). Efectos de una intervención siguiendo el modelo enfocado en la familia para promover avances en el desarrollo del lenguaje de los niños. Revista de Investigación en Logopedia, $\quad 8 \quad$ (2), 165-181. $\quad$ Recuperado de https://revistas.ucm.es/index.php/RLOG/article/view/58734/4564456548442

Duárez, S. (2019). "Habilidades sociales para mejorar la convivencia escolar en estudiantes de la Institución Educativa "Antonio Raimondi Dell Acqua” - Saltur" (tesis de Posgrado). Universidad Cesar Vallejo, Perú. 
Fernández, J. (2015). Convivencia escolar. Un estudio en la provincia de Sevilla (España). International Journal of Educational Research and Innovation (IJERI), 3, 175-191.

Flores, G. (2018). Influencia del programa "Entrenando mis habilidades sociales" en la convivencia escolar en estudiantes de sexto grado de primaria. (Tesis de Pregrado). Universidad San Martin de Porres, Perú.

Gómez, E. y Muñoz, M. (2014). Escala de Parentalidad Positiva. Manual. Recuperado de http://www.observaderechos.cl/site/wp-content/uploads/2013/12/Manual-de-la-Escala-de-ParentalidadPositiva.pdf

Gómez, M. (2017). Alternativa Educativa para favorecer el desarrollo de la comunicación oral en niños de preescolar, atendidos por el programa "Educa a tu Hijo" en la Comunidad "La Aduana" (Tesis de Pregrado). Instituto Superior de Cultura Fisica Manuel Fajardo Holguin, Cuba.

González, E. (2018). "La atención parental en el desarrollo del lenguaje infantil de niños y niñas de 1 a 3 años de la modalidad creciendo con nuestros hijos". (Tesis de postgrado). Universidad técnica de Ambato, Ecuador.

Guevara, G. (1996). “La relación familia-escuela”. Educación 2001, 9, 6-13.

Hernández-Sampieri y Mendoza, C. (2018). Research Methodology. Quantitative, qualitative and mixed routes. Mexico: Mc Graw-Hill

Jiménez, E. (2019). Implementación de estrategias didácticas desde el enfoque comunicativo para el desarrollo de la comunicación oral, en el contexto del Reajuste Curricular 2016 (Tesis de Pregrado). Universidad Central del Ecuador, Ecuador.

López-Roldán, P y Fachelli, S. (2015). Metodología de la Investigación Cuantitativa. Recuperado de https://ddd.uab.cat/pub/caplli/2016/163567/metinvsoccua_a2016_cap2-3.pdf

Luna, C., Montealegre L., y Orozco S. (2019). Vínculo comunicativo: una mirada fonoaudio lógica a través de la percepción de la madre adolescente. Revista PALIMPSESTO, 9 (16), 107-121. Recuperado de http://revistas.usach.cl/ojs/index.php/palimpsesto/article/view/pa.v9i16.3920/26003179

Manriquez, M. S. (2014). Convivencia y clima escolar convivencia y clima escolar conocimiento. Ultima década $n^{\circ} 41$, Proyecto Juventudes, 153-158.

Minedu (2016). Currículo Nacional de la Educación Básica. Recuperado de http://www.minedu.gob.pe/curriculo/pdf/curriculo-nacional-2016-2.pdf

Ministerio de Educación. (2017). Programa curricular de educación primaria. Recuperado de http://www.minedu.gob.pe/curriculo/pdf/programa-curricular-educacion-primaria.pdf 
Ministerio de Educación. (2018). ¿Qué aprendizajes logran nuestros estudiantes? Recuperado de http://umc.minedu.gob.pe/wp-content/uploads/2018/10/Informe-Nacional-ECE-2018.pdf

Ministerio de Educación. (2018). Lineamientos para la gestión de la convivencia escolar, la prevención y la atención de la violencia contra niñas, niños y adolescentes. Recuperado de http://repositorio.minedu.gob.pe/bitstream/handle/MINEDU/6088/Lineamientos\%20para\%20la\%20gest i\%C3\%B3n\%20de\%20la\%20convivencia\%20escolar\%2C\%20la\%20prevenci\%C3\%B3n\%20y\%20la\% 20atenci\%C3\%B3n\%20de\%20la\%20violencia\%20contra\%20ni\%C3\%B1as\%2C\%20ni\%C3\%B1os\%20 y\%20adolescentes.pdf?sequence $=1 \&$ isAllowed $=\mathrm{y}$

Morales, D. y Rodríguez, J. (2017). El recreo: un camino hacia la prevención de comportamientos agresivos. Inclusión y desarrollo, 4(2), 29-39.

Navarro, C. (2018). Influencia Familiar en la expresión oral de los estudiantes (Tesis de pregrado). Universidad nacional de Piura, Perú.

Palomino, M. (2017). Programa de sensibilización parental y su influencia en el área de comunicación de los estudiantes de la Institución Educativa $N^{o} 31398$ de Huancavelica - 2017 (Tesis de Posgrado). Universidad Cesar Vallejo, Perú.

Pérez, L. y Santillán, M. (2018). Estilos de crianza parental en la convivencia escolar en niños de 5 a 6 años (Tesis de Posgrado). Universidad de Guayaquil, Ecuador.

Pérez, P. y Salmerón, T. (2006). Desarrollo de la comunicación y del lenguaje: indicadores de preocupación. Revista Pediatría Atención Primaria, 8(32), 111-125. Recuperado de https://www.redalyc.org/pdf/3666/366638693012.pdf

Ramírez, J. (2002). La expresión oral. Contextos Educativos, 5, 57-72. Recuperado de https://dialnet.unirioja.es/descarga/articulo/498271.pdf

Sandoval, M. (2014). Convivencia y clima escolar: claves 160 de la gestión del conocimiento. Última década, 153-178.

Saneleuteiro, E., y López-García-Torres, R. (2017). Violencia escolar: derechos y deberes para la convivencia. Tendencias Pedagógicas, 30, 267-280.

Suárez, C. (2017). Influencia del rol paterno en la calidad de convivencia escolar en niños de 4 a 5 años (Tesis de Pregrado). Universidad de Guayaquil Facultad de Filosofía, Letras y Ciencias de la Educación, Ecuador.

Suastegui, O. y Mojica, S. (2019). El entorno familiar en la convivencia escolar. Talleres De valores humanos para padres de familia (Tesis de Posgrado). Universidad de Guayaquil, Ecuador. 
Suybate, M. (2019). Nivel de lenguaje oral en niños y niñas de 5 años de una institución educativa del distrito de Ventanilla (Tesis de postgrado). Universidad San Ignacio De Loyola, Perú.

Torán, M. (2016). La narrativa oral como elemento favorecedor del desarrollo oral del vocabulario en la etapa 3-6 años (Tesis de doctorado). Recuperado de https://bit.ly/2UedjBL

UNICEF (2020) Mamás y papás deben apoyar el aprendizaje de las y los adolescentes en el hogar. Recuperado de: https://www.unicef.org/bolivia/historias/mam\%C3\%A1s-y-pap\%C3\%A1s-debenapoyar-el-aprendizaje-de-las-y-los-adolescentes-en-el-hogar

BBC NEWS (2020) Cuarentena por coronavirus: qué es el "efecto padres" en la educación y cómo podría marcar a la generación covid-19. Recuperado de: https://www.bbc.com/mundo/noticias52636384

Vargas, N., Morales, M., Witto, A., Zamorano, J., Olhaberry, M., Farkas, C. (2016) ¿En qué medida la mentalización parental y el nivel socioeconómico predicen el lenguaje infantil? PSICOPERSPECTIVAS, 1(1), 169. Recuperado de http://psicoperspectivas.cl/index.php/psicoperspectivas/article/view/690

Velásquez, M. (2019). La sobreprotección de los padres en el desarrollo social de los niños y niñas de tres a cinco años de edad del C.E.P. Santa Úrsula -2014 (tesis de pregrado). Recuperado de http://repositorio.unjfsc.edu.pe/bitstream/handle/UNJFSC/3682/tesis\%20final.pdf?sequence=1\&isAllow ed $=\mathrm{y}$ 